\title{
Conhecimento etnoveterinária dos produtores rurais dos municípios de Cacoal e Espigão D'Oeste/Rondônia
}

Objetivo deste trabalho foi identificar e analisar o conhecimento etnoveterinária praticado em pequenas propriedades rurais localizadas nos municípios de Cacoa e Espigão D'Oeste/Rondônia. Para tal, realizaram-se visitas a 82 propriedades de agricultura familiar, para entrevistar os proprietários sobre as espécies medicinais usadas, formas de armazenamento, de uso, parte da planta e espécie animal tratada, assim como a origem do conhecimento etnobotânico e etnofarmacológico por meio de um questionário semiestruturado com questões fechadas e abertas. Do total de propriedades visitas, 54 relataram o uso frequente de plantas medicinais em animais, sendo identificadas 24 espécies para tratar nove diferentes patologias. Os bovinos foram a espécie com maior número de relato de tratamento, seguidos dos cães, aves e equinos. A principal via de administração utilizada foi por via oral por meio do chá de folhas, cascas e/ou raízes, ou misturados ao alimento. Neste estudo, observou-se que o conhecimento etnoveterinário é adquirido principalmente pela experiência trazida pelas gerações mais antigas, motivado, também, pela redução da contaminação do alimento por resíduos de medicamentos e baixo custo de tratamento. Das plantas citadas pelos entrevistados, se destacaram com maior valor de uso a copaíba, nim, melão-de-são-caetano e arnica, demonstrando assim o comum emprego desta prática, principalmente apoiada no resgate do conhecimento tradicional trazido pelos migrantes vindos de outras regiões do Brasil, com a cultura local.

\section{Ethnoveterinary knowledge of rural producers in the city of Cacoal and Espigão do D'Oeste/Rondônia}

\begin{abstract}
The objective of this work was to identify and analyze the ethnoveterinary knowledge in small rural properties in the municipalities of Cacoal and Espigão D'Oeste in Rondônia, Brazil. 82 small rural family farms were visited, and a semi-structured questionnaire was applied to them. The questionnaire was composed of multiplechoice and open questions related to which medicinal plant species used, storage and use methods, part of the plant used, animal species treated, the ethnobotanical origin, and ethnopharmacological knowledge. From all the farms visited, 54 farms reported frequent use of medicinal plants in animals. The study identified 24 medicinal plant species being used to treat 9 different pathologies. Cattle presented the highest number of treatment reports, followed by dogs, birds, and horses. The most used route of administration was oral through leaf, bark, and/or root tea or mixed with food. In this study, it was observed that ethnoveterinary knowledge is acquired mainly by the experience passed down through older generations. Also, ethnoveterinary knowledge use is motivated by the reduction of food contamination by medication residues and the low cost of treatment. For use, the most valuable plants mentioned by the interviewees were the copaiba, neem, bitter melon, and mountain arnica. This study has shown the common use of ethnoveterinary knowledge, this practice mainly supports the rescue of the traditional knowledge brought by migrants from other regions of Brazil.
\end{abstract}

Keywords: Medicinal plants; Amazon; Treatment; Production animals.

Sandro Vargas Schons (iD

Universidade Federal de Rondônia, Brasil http://lattes.cnpq.br/2469714239413847 http://orcid.org/0000-0001-9811-5356 sandroschons@unir.br

Kaisa Freitas Araujo in

Universidade Federal de Rondônia, Brasil http://lattes.cnpq.br/5919913585422782 http://orcid.org/0000-0001-9834-3594 araujokaisa@gmail.com

\section{Átila Bezerra Mira (D)}

Universidade Federal de Rondônia, Brasil http://lattes.cnpq.br/8487826290510262 http://orcid.org/0000-0002-8956-0965 atilaabm4@gmail.com
Mariana Ferraz Rodrigues

Universidade Federal de Rondônia, Brasil

marianaferrazadv@gmail.com

Ezequiel Ferreira Barbosa

Universidade Federal de Rondônia, Brasil http://lattes.cnpq.br/5495063828463883

http://orcid.org/0000-0002-5371-6366 ezequielbarbos@gmail.com

Kelly Cristina Barbosa (iv

Universidade Federal de Rondônia, Brasil

http://lattes.cnpq.br/3676694028684236

http://orcid.org/0000-0001-7131-3504

kellybarbosa.mv@gmail.com
Francisco Carlos da Silva

Universidade Federal de Rondônia, Brasi http://lattes.cnpq.br/7680005664798361 http://orcid.org/0000-0003-4105-3806 fcsbiologicalscience@gmail.com
DOI: 10.6008/CBPC2179-6858.2020.004.0036
Referencing this:

SCHONS, S. V.; ARAUJO, K. F.; MIRA, Á. B.; RODRIGUES, M. F.; BARBOSA, E. F.; BARBOSA, K. C.; SILVA, F. C.. Conhecimento etnoveterinária dos produtores rurais dos municípios de Cacoal e Espigão D'Oeste/Rondônia. Revista Ibero-Americana de Ciências Ambientais, v.11, n.4, p.432-440, 2020. DOI:

http://doi.org/10.6008/CBPC2179-6858.2020.004.0036 


\section{INTRODUÇÃO}

As plantas medicinais têm sido utilizadas no tratamento de inúmeras enfermidades de humanos e animais, desde os tempos ancestrais, e trouxeram consigo elementos tradicionais, vindos de heranças dos antepassados, passados através das observações e práticas terapêuticas. Atualmente, o uso de tais plantas está sendo incentivado na medicina veterinária, principalmente, pela redução de efeitos colaterais, menor contaminação por resíduo em produtos de origem animal e menor custo com tratamento (GURIB-FAKIM, 2006).

Contudo, no Brasil, o uso dessas plantas na clínica médica veterinária é ainda incipiente, principalmente pelo baixo número de estudos e divulgação das pesquisas, da diversidade de espécies com as quais os veterinários tratam, além de preconceitos quanto ao uso de chás caseiros. Desse modo, é necessário que o conhecimento popular seja aplicado às teorias e práticas, direcionadas à promoção da saúde animal, empregando plantas medicinais no tratamento e cura de enfermidades, em determinada população animal, o que caracteriza o estudo etinoveterinária (MCCORKLE, 1986; TEIXEIRA et al., 2015).

A etnoveterinária, portanto, desempenha um papel importante na conservação de conhecimentos tradicionais, principalmente sobre plantas medicinais usadas no tratamento de doenças em animais de companhia e de produção (BULLITTA et al., 2018). Para Monteiro et al. (2012), o uso dessas plantas está ligado essencialmente às influências culturais indígenas, africanas e europeias.

Na Amazônia, o uso de tais plantas guarda elementos de diferentes culturas, caracterizadas na construção de tradições de uso inventadas a partir de conhecimentos e saberes oriundos de diferentes povos. Os diferentes modos de vida configurados a partir da colonização, desenvolveram conhecimentos que mantém nexos com o passado, e incluem práticas ancestrais em seus principais elementos (SANTOS, 2000). De acordo com Aires (2013), nesta região, o uso medicinal de produtos derivados das plantas, por comunidades locais, continua a ser uma prática significativa. Este fato ocorre devido à relação harmoniosa entre o conhecimento etnobotânico da biodiversidade da Floresta Amazônica e o homem, ao longo da história, com a observações de fenômenos naturais e experiências com os recursos disponíveis, o que resultou em um vasto conhecimento empírico sobre uso de tais plantas, passados por gerações (TORRES et al., 2010). Com base no exposto, o objetivo deste trabalho foi identificar e analisar o conhecimento etnoveterinário praticado em pequenas propriedades rurais de áreas antropizadas da Floresta Amazônica, para a implantação da agropecuária.

\section{MATERIAIS E MÉTODOS}

Realizaram-se visitas a pequenas propriedades rurais agrícola-familiares pertencentes aos municípios de Cacoal e Espigão D'Oeste/Rondônia, para coleta de dados sobre o uso de plantas medicinais em animais. A região em estudo se localiza na Amazônia Ocidental e é formada por áreas antropizadas da Floresta Amazônica para a implantação da agropecuária. Os municípios de Cacoal e Espigão D’Oeste foram criados a partir do projeto do Instituto Nacional de Colonização e Reforma Agrária/INCRA, que instalou no Estado uma 
série de subprojetos integrados de colonização e ocupação de áreas florestais, por colonos vindos da Região Sul e Sudeste. Durante o período de estudo, a região possuía um total de 6.331 estabelecimentos agropecuários, ocupando uma área geográfica de 480.876 hectares (IBGE, 2006). Porém, atualmente o número de propriedades teve uma redução para 5.814, ocupando a mesma área geográfica (IBGE, 2017), distinguida na Figura 1.

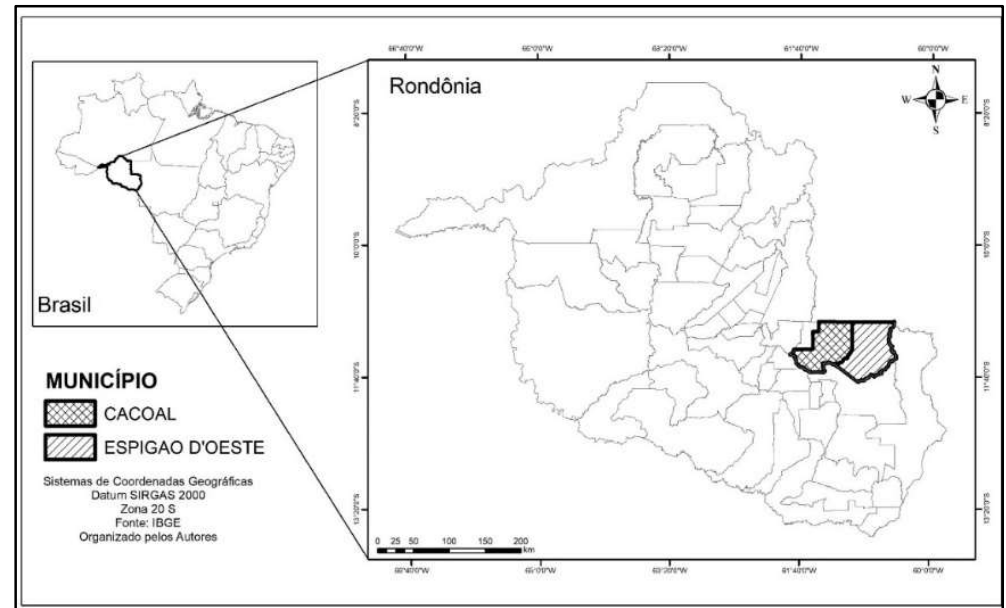

Figura 1: Localização da região de estudo, nos municípios de Cacoal e Espigão D’Oeste, em Rondônia.

A coleta de dados foi realizada entre os meses de março e junho de 2014 , durante visita a 82 propriedades rurais. Para tal, foi utilizado um questionário semiestruturado com questões fechadas e abertas, relacionadas aos motivos de uso de plantas medicinais em animais, forma de coleta das plantas, identificação e armazenamento destas, bem como quais partes eram utilizadas e a forma de administração em diferentes espécies animais. Antes de responder aos questionários, todos os entrevistados foram informados dos objetivos do trabalho e após o consentimento, as entrevistas foram realizadas. Os resultados foram inseridos na base de dados do Epi Info ${ }^{\mathrm{TM}} 7.2 .1$ e foram tabulados e analisados pelo método quantitativo.

Para calcular o valor de uso de uma espécie por entrevistado, foi utilizada a fórmula UVis = $\Sigma$ Usi / nis, no qual Usi corresponde o número de uso da planta medicinal e nis o número de entrevista com o mesmo produtor, contudo, para este estudo nis foi considerado um (01), uma única entrevista. Para calcular o valor de uso das espécies citadas pelos entrevistados foi utilizada a fórmula UVs = $\Sigma$ UVsi / $n$, sendo UVsi o número vezes de uso da planta e o $n$ o número de entrevistados que relataram a utilização (MONTEIRO et al., 2011). As demais variáveis foram analisadas através da estatística descritiva com distribuição de frequência conforme Wondimu et al. (2007).

As indicações terapêuticas das plantas medicinais utilizada na saúde animal, foram agrupadas em nove condições clínicas: infecções (If), cicatrização de feridas (Cf), cólica abdominal (Ca), anti-inflamatório (Ai), problemas no parto (Ppr), antidiarreico (Di), picada de cobra (Pc), ectoparasitoses (Ep) e anti-helmínticos (Ah). 


\section{RESULTADOS}

Realizaram-se visitas a 47 propriedades rurais do município de Cacoal $(57,4 \%)$ e 35 de Espigão do Oeste $(42,6 \%)$, totalizando 82 . Todas as propriedades visitadas relataram a prática do uso de plantas medicinais, porém, em $65 \%$ dessas, a prática ocorria em animais e humanos, e $35 \%$ somente para tratar patologias humanas.

Entre os animais tratados, os bovinos foram os de maior ocorrência, com 60,6\%, seguidos dos cães, aves e equinos, com $21,2 \%, 15,2 \%$ e 3,1\%, respectivamente. Foram identificadas 24 espécies de plantas medicinais utilizadas na saúde animal e um produto ecológico, sendo administrados na forma de infusão de folhas, raízes e cascas, misturadas a água ou via beberagem (62,3\%), além, da administração de raspas ou macerados de cascas, frutos e folhas misturadas a comida (17,0\%). O uso de banho terapêutico foi mencionado em $15,1 \%$ das propriedades visitadas (tabela 1 ).

As plantas medicinais mencionadas durante as entrevistas eram usadas no tratamento de nove (09) diferentes condições clínicas, sendo o maior número de espécies indicada para o tratamento de infecções $(18,8 \%)$, sucessivamente, como anti-inflamatórios e antidiarreicos $(15,6 \%)$, no controle das ectoparasitoses, como carrapatos, piolhos e pulgas, e em cólicas abdominais $(12,5 \%)$. As demais patologias tratadas por número de espécimes de plantas mencionadas estão descritas na tabela 2 . 0 valor de uso, a forma de administração e a espécie animal tratada estão descritas na tabela 1.

Conforme os produtores, os principais motivos que levam ao uso de plantas medicinais em animais foram; a preocupação com resíduos deixados nos alimentos de origem animal $(15,6 \%)$, menor custo com tratamento $(6,0 \%)$ e resultados insatisfatórios com tratamento tradicional $(1,2 \%)$. Porém, $68,6 \%$ dos entrevistados, mencionaram que era uma prática passada por gerações. Ao mesmo tempo, $87,8 \%$ informavam-se, de novas plantas a serem usadas na saúde animal com parentes, seguido de $8,2 \%$, com profissionais da área e 6,1\% através da leitura de jornais, revistas e programas de televisão. Quanto ao resultado do tratamento com plantas medicinais, 65,0 \% dos entrevistados relataram estarem satisfeitos, $25,5 \%$ muito satisfeitos e $6,0 \%$ insatisfeitos. Conforme os produtores, não foram observados sinais clínicos sugestivos de intoxicações ou efeitos colaterais após o tratamento dos animais com as espécies medicinais.

Tabela 1: Plantas medicinais, valor de uso das espécies, parte da planta, forma de administração e espécie animal.

\begin{tabular}{lllllll}
\hline $\begin{array}{l}\text { Nome } \\
\text { Popular }\end{array}$ & Nome Científico & Uvsi & Uvs & Uso & Forma de uso & $\begin{array}{l}\text { Espécie } \\
\text { Animal }\end{array}$ \\
\hline Algodoeiro & Gossypium herbaceum & 1 & 0.019 & Folhas & Infusão & $\begin{array}{l}\text { Bovino } \\
\text { Ave }\end{array}$ \\
Alho & Allium sativum & 3 & 0,058 & Bulbo & Macerado & $\begin{array}{l}\text { Bovino } \\
\text { Bovino }\end{array}$ \\
Amoreira & Morus nigra & 1 & 0,019 & Folhas & Infusão & Bovino \\
Arnica & Arnica montana & 4 & 0,784 & Folhas & Tópico & Cão \\
Babaçu & Orbignya phalerata & 1 & 0,019 & Casca & Moído & Bovino \\
Boldo & Peumus boldus & 1 & 0,019 & Folhas & Infusão & Cão \\
$\begin{array}{l}\text { Cajueiro } \\
\text { Roxo }\end{array}$ & Anacardium occidentale & 3 & 0,058 Casca/ & Folhas & Infusão & Bovino \\
Cedro & Cedrus spp. & 1 & 0,019 & Casca & Infusão & Bovino \\
Coentro & Coriandrum sativum & 1 & 0,019 & Semente & Infusão & Bovino \\
Corama & Bryophyllum pinnatum & 1 & 0,019 & Folhas & Infusão & Cão \\
Copaíba & Copaifera spp. & 6 & 0,117 & Óleo & Tópico & Bovino \\
& & & & & Ave
\end{tabular}




\begin{tabular}{|c|c|c|c|c|c|c|}
\hline & & & & & & Cão \\
\hline Curindiba & Piptocarpha rotundifolia & 1 & 0,019 & Casca & Infusão & Bovinos \\
\hline Guiné & Petiveria alliacea & 1 & 0,019 & Raiz & Infusão & $\begin{array}{l}\text { Bovino } \\
\text { Equino }\end{array}$ \\
\hline $\begin{array}{l}\text { Melão-de-são- } \\
\text { Caetano }\end{array}$ & Momordica charantia & 4 & 0,784 & $\begin{array}{l}\text { Folhas/ } \\
\text { Raiz }\end{array}$ & Infusão & $\begin{array}{l}\text { Bovino } \\
\text { Ave }\end{array}$ \\
\hline $\mathrm{Nim}$ & Azadirachta indica & 6 & 0,117 & $\begin{array}{l}\text { Folhas/ } \\
\text { Fruto }\end{array}$ & $\begin{array}{l}\text { Banho/ } \\
\text { Infusão }\end{array}$ & Bovino \\
\hline Pau alho & $\begin{array}{l}\text { Gallesia integrifolia (Spreng.) } \\
\text { Harms }\end{array}$ & 2 & 0,039 & $\begin{array}{l}\text { Casca } \\
\text { Folhas }\end{array}$ & Banho & $\begin{array}{l}\text { Cão } \\
\text { Ave }\end{array}$ \\
\hline Pau pereira & Platycyamus regnellii & 1 & 0,019 & Casca & Banho & Ave \\
\hline Própolis & & 1 & 0,019 & Resina & Xarope & Bovino \\
\hline Assa-peixe & Vernonia polysphaera & 1 & 0,019 & Folhas & Tópico & Cão \\
\hline Açafrão & Crocus sativus & 1 & 0,019 & Folhas & Banho & Bovino \\
\hline Jalapa & Mirabilis jalapa & 2 & 0,039 & $\begin{array}{l}\text { Folhas/ } \\
\text { Raízes }\end{array}$ & Raspa & $\begin{array}{l}\text { Cão } \\
\text { Bovino }\end{array}$ \\
\hline Cabelo de milho & Zea mays. L & 3 & 0,058 & -- & Raspas & Bovinos \\
\hline Hortelão & Mentha spicata. L. & 1 & 0,019 & Folhas & Banho & Bovino \\
\hline Coração da bananeira & Musa paradisíaca & 2 & 0,039 & $\begin{array}{l}\text { Umbigo } \\
\text { banana }\end{array}$ & Infusão & Bovino \\
\hline Batata doce & Ipomoea batatas & 1 & 0,019 & Folhas & Infusão & Bovino \\
\hline
\end{tabular}

Tabela 2: Frequência absoluta e porcentagem das indicações terapêuticas das plantas medicinais identificadas no tratamento de animais.

\begin{tabular}{lll}
\hline Indicação terapêutica & Número Absoluto & Porcentagem \\
\hline Infecções & 6 & $18,8 \%$ \\
Cicatrização & 3 & $9,4 \%$ \\
Cólica abdominal & 4 & $12,5 \%$ \\
Anti-inflamatório & 5 & $15,6 \%$ \\
Problemas no parto & 1 & $3,1 \%$ \\
Antidiarreico & 5 & $15,6 \%$ \\
Picada de cobra & 2 & $6,2 \%$ \\
Ectoparasitoses & 4 & $12,5 \%$ \\
Anti-helmíntico & 2 & $6,25 \%$ \\
\hline
\end{tabular}

\section{DISCUSSÃO}

O uso de plantas medicinais na saúde e produção animal foi observada em $65 \%$ das propriedades rurais visitas, especialmente no tratamento de bovinos. A maior frequência nesta espécie, está associado, principalmente, ao alto custo de produção, ao preço dos medicamentos, bem como a higienização dos equipamentos, utensílios e instalações, levando os produtores a buscarem alternativas para reduzir os custos com controle das enfermidades e melhorar a qualidade do produto da propriedade (ARCEGO, 2005). Entretanto, neste estudo, as tradições deixadas pelas gerações passadas foram os principais motivos que levaram os produtores a praticar o uso de plantas medicinais em animais. De acordo com Oliveira et al. (2009) essa forma de conhecimento se dá pela transmissão de sabedoria natural, através das suas práticas, por meio da observação de outra pessoa, e posterior aprendizado que é repassado. A transmissão oral é o principal veículo de perpetuação desse conhecimento tradicional, ou melhor, milenar, o qual acontece no cotidiano das comunidades, ou seja, através de um contato constante entre os mais velhos e os mais jovens (PILLA et al., 2006).

Estudos etnoveterinários tem sido descritos em diversas regiões do Brasil, entre esses, na comunidade de Igrejinha/Minas Gerais (DIAS et al., 2014), comunidade de Várzea Comprida Oliveira do município de Pombal/Paraíba (ANDRADE et al., 2012), assentamento rural de Seropédica/Rio de Janeiro (SILVA et al., 2014), em Ivaiporã/Paraná (SOUZA et al., 2015), Patos/Paraíba (MARINHO et al., 2007), 
Jataí/Goiás (VIU et al., 2011), Ilha de Marajó/Pará (MONTEIRO et al., 2011) e entre discentes do curso de medicina veterinária da Escola Superior de Agricultura de Mossoró/Rio Grande do Norte (ALMEIDA et al., 2006). No entanto, este estudo é o primeiro realizado no estado de Rondônia, localizado na Amazônia Ocidental, e ocupada por áreas antropizadas da Floresta Amazônica para implantação da agropecuária, bem como por diferentes modos de vida, configurados a partir da colonização por emigrantes oriundos de outras regiões do país.

Neste estudo foram identificadas 24 espécies medicinais, sendo quatro com maior número de uso: o nim (Azadirachta indica) (UVs=0,117), a copaíba (Copaifera spp.) (UVs=0,117), o melão-de-são-caetano (Momordica charantia) (UVs=0,078) e a arnica (Arnica montana) (UVs=0,078). O nim, também foi mencionada em levantamentos realizados na llha do Marajó (MONTEIRO et al., 2011), e em comunidades rurais do município de Araçuaí/MG (DIAS et al., 2014), e em ambas utilizada como repelente.

Estudos realizados com extrato de semente de nim e óleo emulsionável, em concentrações de $2 \%$, demonstram significativo potencial no controle do carrapato bovino, pois ocasionam a mortalidade nos primeiros dias após o tratamento e interferem na reprodução, mostrando ser uma alternativa aos carrapaticidas normalmente utilizados (BROGLIO-MICHELETTI et al., 2010). Outra planta citada no controle das ectoparasitoses foi o pau alho (Gallesia integrifólia), com valor de uso de 0,039, contudo, essa espécie foi mencionada no tratamento de carrapatos, pulgas e piolhos de cães e aves. $O$ uso do pau alho também foi relatado em um estudo etnoveterinário realizado em assentamentos rurais de Seropédica/RJ, para controle de carrapatos e pulgas de galináceos e equinos (SILVA et al., 2014). O pau pereira (Platycyamus regnellii) e o açafrão (Crocus sativus) também foram citados pelos produtores de Rondônia, sendo utilizados como repelente em bovinos e galináceos. Conforme Oliveira et al. (2009), os produtores utilizam tais plantas como terapia alternativa para o controle de verminoses, o que ocasiona uma redução de custos vindos da compra de anti-helmínticos, além de controlar as endoparasitoses e as ectoparasitoses, como carrapatos e moscas, resultando em um ganho de produtividade significativo.

Quanto as indicações terapêuticas na prática de cura ou de forma profilática, as plantas com ação nas infecções foram as mais citadas entre os entrevistados (Tabela 2), e citam para tal: a copaíba (Copaifera spp.), algodoeiro (Gossypium Herbaceum), amoreira (Morus Nigra), corama (Bryophyllum pinnatum), açafrão (Crocus sativus) e melão-de-são-caetano (Momordica charantia). Entre essas, a Copaifera spp., teve o maior número de uso (UVs= 0,117 ), semelhante ao encontrado em levantamento etnoveterinário realizados na Ilha de Marajó, na qual, essa foi a segunda mais citada (MONTEIRO et al.,2011). Da copaibeira é extraído um óleo, denominado óleo de copaíba, que possui inúmeras propriedades medicinais, como ação anti-inflamatória, antimicrobiana e antisséptica (RIGAMONTE-AZEVEDO et al., 2004; PIERI et al., 2009). Trabalhos realizados in vitro também demonstraram ação antimicrobiana frente a Staphylococcus coagulase-positiva multirresistentes isolados de otite canina externa (ZIECH et al., 2013).

Contudo, estas espécies foram mencionadas para tratar grandes animais, com exceção corama, utilizada em pequenos animais, os cães. A Bryophyllum pinnatum, também conhecida popularmente como "folha-da-fortuna" tem sido largamente utilizada na medicina tradicional, principalmente para o tratamento 
de inflamações, infecções, feridas, ulcerações e gastrite (OKWU et al., 2011).

A copaíba, além de ser indicada nas infecções, também foi mencionada no tratamento de cicatrização de feriadas, juntamente como o assa-peixe (Vernonia polysphaera), cedro (Cedrela adorata) e própolis. A copaíba e o cedro são espécies florestais, igualmente indicadas por índios da etnia Xipaya para tratamento de cicatrizações e infecções do trato respiratório superior do homem (SANTOS et al., 2016). Da mesma forma, a Vernonia polyanthes, conhecida popularmente como "assa-peixe", é uma planta típica da Mata Atlântica e tem sido empregada na medicina popular para o tratamento de pneumonia, bronquite, cálculo renal, malária e febre (ROSSATO, 1996).

A ocorrência de doenças em uma população animal, está diretamente relacionada ao manejo, à espécie, à idade, ao clima e às práticas de profilaxia adotadas, sendo proporcionalmente maior o uso de medicamentos nas enfermidades de maior ocorrência na população (BROGLIO-MICHELETTI et al., 2010). Da mesma forma é observado neste estudo, as patologias de origem digestivas, como as diarreias e as cólicas abdominais, de origem parasitária ou infecciosas, foram as com maior valor absoluto de indicações (tabela 2).

Tão logo, os entrevistados mencionaram o uso do cajueiro roxo (Anacardium occidentale), curindiba (Piptocarpha rotundifolia), melão-de-são-caetano (Momordica charantia), cabelo de milho (Zea mays. L) e o coração da banana (Musa paradisíaca) como antidiarreico, e a arnica (Arnica Montana), boldo (Peumus boldus) e coentro (Coriandrum sativum) para tratar as cólicas abdominais. Por outro lado, levantamentos realizados em Mossoró/RN (ALMEIDA et al., 2006), na Ilha de Marajó/Pará (MONTEIRO et al., 2011), e Jataí/ Goiás (VIU et al., 2011) tiveram maior número de espécies medicinais indicadas para o tratamento das cicatrizações de feridas, e em Seropédica/ Rio de Janeiro (SILVA et al., 2014), para o controle anti-helmíntico. Possivelmente, as condições climáticas do estado de Rondônia, caracterizado pelo clima tropical, criem um microclima que favoreçam a perpetuação dos ectoparasitas no ambiente, bem como patógenos oportunistas responsáveis por transtornos digestivos, elevando assim, maiores indicações de plantas para estas patologias.

Chama a atenção neste estudo, a utilização do coentro (Coriandrum sativum) e o guiné (Petiveria alliacea) para o tratamento de picada de cobra. Conforme Mors et al. (2000), existem várias espécies da flora brasileira com potencial antiofídico, que podem ser utilizadas no futuro como drogas ou modelos para construção de novas drogas. A avaliação terapêutica de tais plantas in natura ou in vitro, principalmente de seus constituintes, tais como flavonoides, alcaloides, triterpenos, sesquiterpenos e ligninas são de grande importância para possível tratamento dos acidentes ofídicos (MORS et al., 2000), além de terem atividades farmacológicas na complementação da soroterapia tradicional, principalmente no uso de animais domésticos ou de interesse comercial, uma vez que, para estes, o soro tradicional não é disponibilizado (SOARES et., 2005).

\section{CONCLUSÕES}

Diante disso, o levantamento da biodiversidade botânica utilizada no tratamento de animais, em 
propriedades rurais dos municípios de Cacoal e Espigão D'Oeste, demonstra o comum emprego desta prática, principalmente apoiado no resgate do conhecimento tradicional trazido pelos migrantes, vindos de outras regiões do Brasil, sobre as indicações terapêuticas e o uso das espécies vegetais. Dentre as enfermidades mais tratadas, destacam-se as ectoparasitoses e os transtornos digestivos, e o uso maior nestas patologias está associado ao clima da região, tropical, que favorece o crescimento e manutenção dos agentes etiológicos no ambiente. Conduto, na prática veterinária, estes medicamentos naturais são pouco utilizados, principalmente, pelo desconhecimento dos mecanismos de ação, formas de uso e dose terapêutica usadas por espécie animal, sendo necessário a realização de ensaios clínicos, toxicológicos e químicos para comprovar tais propriedades farmacológicas.

\section{REFERÊNCIAS}

AIRES, W. C.. Estudo fitoquímico de Himatanthus sucuuba na busca de substâncias com atividades biológicas úteis. In: SEMINÁRIO DE INICIAÇÃO CIENTÍFICA DA UFPA, 24. Anais. Belém: Faculdade de Química, Instituto de Ciências Exatas e Naturais, 2013.

ALMEIDA, K. S.; FREITAS, F. L. C.. Etnoveterinária: a fitoterapia na visão do futuro profissional veterinário. Revista Verde de Agroecologia e Desenvolvimento Sustentável, v.1, n.1, p.67-74, Mossoró, 2006.

ANDRADE, S. E. O.; MARACAJÁ, P.; SILVA, R. A.; FREIRES, G F.; PEREIRA, A. M.. Estudo etnoveterinário de plantas medicinais na comunidade Várzea Comprida dos Oliveiras, Pombal, Paraíba, Brasil. Revista Verde de Agroecologia e Desenvolvimento Sustentável, Mossoró, v.7, n.2, p.193-198, 2012.

ARCEGO, M. S. C.. Plantas medicinais no controle de doenças no gado leiteiro. São João da Urtiga: ASCAR, 2005.

BROGLIO-MICHELETTI, S. M. F.; VALENTE, E. C. N.; SOUZA, L. A.; ARAÚJO, A. M. N.. Extratos de plantas no controle de Rhipicephalus (Boophilus) microplus (Canestrini, 1887) (Acari: Ixodidae) em laboratório. Revista Brasileira de Parasitologia Veterinária, v.18, n.4, p.44-48, 2009. DOI: http://doi.org/10.4322/rbpv.01804008

BULLITTA, S.; RE, G. A.; MANUNTA, M. D. I.; PILUZZA, G.. Traditional knowledge about plant, animal, and mineralbased remedies to treat cattle, pigs, horses, and other domestic animals in the Mediterranean island of Sardinia. Journal of Ethnobiology and Ethnomedicine, v.14, n.50, 2018. DOI: https://doi.org/10.1186/s13002-018-0250-7

DIAS, I. S.; SANTOS, R. A.. A etnoveterinária como ferramenta agroecológica na produção animal. In: FÓRUM DE ENSINO, PESQUISA, EXTENSÃO E GESTÃO, 8. Anais. Salvador: FEPEG, 2014.

GURIB-FAKIM, A.. Medicinal plants: traditions of yesterday and drugs of tomorrow. Molecular Aspects of Medicine, v.27, n.1, p.1-93, 2006. DOI: https://doi.org/10.1016/i.mam.2005.07.008

IBGE. Instituto Brasileiro de Geografia e Estatística. Censo agropecuário 2006. Rio de Janeiro: IBGE, 2006.
IBGE. Instituto Brasileiro de Geografia e Estatística. Censo agropecuário 2017. Rio de Janeiro: IBGE, 2017.

MARINHO, M. L.. A utilização de plantas medicinais em medicina veterinária: um resgate do saber popular. Revista Brasileira de Plantas Medicinais, Botucatu, v.9, n.3, p.64-69, 2007.

MCCORKLE, C. M.. An introduction to ethnoveterinary research and development. Journal of Ethnobiology, v.6, n.1, p.129-149, 1986.

MONTEIRO, M. V. B.; BEVILAQUA, C. M. L.; PALHA, M. D. C.; BRAGA, R. R.; SCHWANKE, K.; RODRIGUES, S. T.; LAMEIRA, O. A.. Ethnoveterinary knowledge of the inhabitants of Marajó Island, Eastern Amazônia, Brazil. Acta Amazônica, Manaus, v.41, n.2, p.233-242, 2011. DOI: https://doi.org/10.1590/S0044-59672011000200007

MONTEIRO, M. V. B.; RODRIGUES, S. T.; CAMURÇAVASCONCELOS, A. L. F.. Plantas medicinais utilizadas na medicina etnoveterinária praticada na Ilha do Marajó. Belém: Embrapa Amazônia Oriental, 2012.

MORS, W. B.; NASCIMENTO, M. C.; PEREIRA, B. M. R.; PEREIRA, N. A.. Plant natural products active against snake bite - the molecular approach. Phytochemistry, v.55, n.6, p.627-642, 2000. DOI: https://doi.org/10.1016/S00319422(00)00229-6

OKWU, D. E.; NNAMDI, F. U.. A novel antimicrobial phenanthrene alkaloid from Bryophyllum pinnatum. EJournal of Chemistry, v.8, n.3, p.1456-1461, 2011. DOI: http://doi.org/10.1155/2011/972359

OLIVEIRA, L. S. T.; SILVA, S. L. C.; TAVARES, D. C.; SANTOS, A. V.; OLIVEIRA, G. C. B.. Uso de plantas medicinais no tratamento de animais. Enciclopédia Biosfera, v.5, n.8, p.18, 2009.

PIERI, F. A.; MUSSI, M. C.; MOREIRA, M. A. S.. Óleo de copaíba (Copaifera sp.): histórico, extração, aplicações industriais e propriedades medicinais. Revista Brasileira de Plantas Medicinais, Botucatu, v.11, n.4, p.465-472, 2009.

PILLA, M. A. C.; AMOROZO, M. C. M.; FURLAN, A.. Obtenção 
e uso das plantas medicinais no distrito de Martim Francisco, Município de Mogi-Mirim, SP, Brasil. Acta Botânica Brasílica, São Paulo, v.20, n.4, p.789-802, 2006. DOI: https://doi.org/10.1590/S0102-33062006000400005

RIGAMONTE-AZEVEDO, O. C.; WADT, P. G. S.; WADT, L. H. O.. Copaíba: ecologia e produção de óleo-resina. Rio Branco: EMBRAPA, 2004

ROSSATO, S. C.. Utilização de plantas por populações do litoral norte do estado de São Paulo. Dissertação (Mestrado em Ciências Biológicas e Botânica) - Universidade de São Paulo, São Paulo, 1996.

SANTOS, F. S. D.. Tradições populares de uso de plantas medicinais na Amazônia. História, Ciências, SaúdeManguinhos, Rio de Janeiro, v.6, n.1, p.919-939, 2000. DOI: https://doi.org/10.1590/S0104-59702000000500009

SANTOS, J. X.; REIS, A. R. S.; MATOS, S. A.; LEÃO, F. M.; CARVALHO, J. C. C.. Caracterização etnobotânica de essências florestais com fins medicinais utilizadas pela Etnia Xipaya, no município de Altamira/PA. Biota Amazônia, v.6, n.2, p.1-8, 2016.

SILVA, W. M. O.; SOUZA, G. F. X. T.; VIEIRA, P. B.; SANAVRIA, A.. Uso popular de plantas medicinais na promoção da saúde animal em assentamentos rurais de Seropédica-RJ. Revista Brasileira Ciência Veterinária, Rio de Janeiro, v.20, n.1, p.3236, 2014. DOI: http://dx.doi.org/10.4322/rbcv.2014.047

SOUZA, A.; SOUZA JÚNIOR, J. B.; TEIXEIRA, E. J. R.; LEN, L. S.; MOURO, G. F.; MONTEIRO, V.. Etnoveterinária na Região de Ivaiporã-PR: Conhecimentos Populares Contribuindo na Construção de Sistemas de Produção Animal Mais
Sustentáveis. Cadernos de Agroecologia, v.9, n.4, 2015.

SOARES, A. M.; MARCUSSI, S.; LOURENÇO, M. V.; JANUÁRIO, A. H.; SAMPAIO, S. V.; GIGLIO, J. R.; LOMONTE, B.; PEREIRA, P. S.. Medicinal plants ith inhibitory properties against snake venoms. Current Medical Chemistry, v.12, p.2625-2641, 2005. DOI: http://dx.doi.org/10.2174/092986705774370655

TEIXEIRA, V. H. S.; FERNANDES, N. C. F.; CORRÊA, F. C.; OLIVEIRA, D. M. C.. Plantas medicinas na etnoveterinária no Brasil - Artigo de revisão. Anclivepa, v.42, n.1, p.926-930, 2015.

TORRES, E. C.; RIBEIRO, A.; MORAES, M. A.. Abordagem fitoquímica e prospecção do potencial antimicrobiano in vitro das partes aéreas de três espécies vegetais pertencentes à família Laminaceae. Educadores dia-a-dia. Curitiba, 2010.

VIU, A. F. M.; VIU, M. A. O. Cerrado e etnoveterinária: o que se sabe em Jataí/GO?. Revista Brasileira de Agroecologia, v.6, n.3, p.49-61, 2011.

WONDIMU, T.; ASFAW, Z.; KELBESSA, E.. Ethnobotanical study of medicinal plants around 'Dheeraa' town, Arsi Zone, Ethiopia. Journal of Ethnopharmacology, v.112, n.1, p.152161, 2007. DOI: http://doi.org/10.1016/j.jep.2007.02.014

ZIECH, R. E.; FARIAS, L. D.; BALZAN, C.; ZIECH, M. F.; HEINZMANN, B. M.; LAMEIRA, O. A.; VARGAS, A. C.. Atividade antimicrobiana do oleorresina de copaíba (Copaifera reticulata) frente a Staphylococcus coagulase positiva isolados de casos de otite em cães. Pesquisa Veterinária Brasileira, v.33, n.7, p.909-913, 2013. DOI: https://doi.org/10.1590/S0100-736X2013000700011

A CBPC - Companhia Brasileira de Produção Científica (CNPJ: 11.221.422/0001-03) detém os direitos materiais desta publicação. Os direitos referem-se à publicação do trabalho em qualquer parte do mundo, incluindo os direitos às renovações, expansões e disseminações da contribuição, bem como outros direitos subsidiários. Todos os trabalhos publicados eletronicamente poderão posteriormente ser publicados em coletâneas impressas sob coordenação da Sustenere Publishing, da Companhia Brasileira de Produção Científica e seus parceiros autorizados. Os (as) autores (as) preservam os direitos autorais, mas não têm permissão para a publicação da contribuição em outro meio, impresso ou digital, em português ou em tradução. 